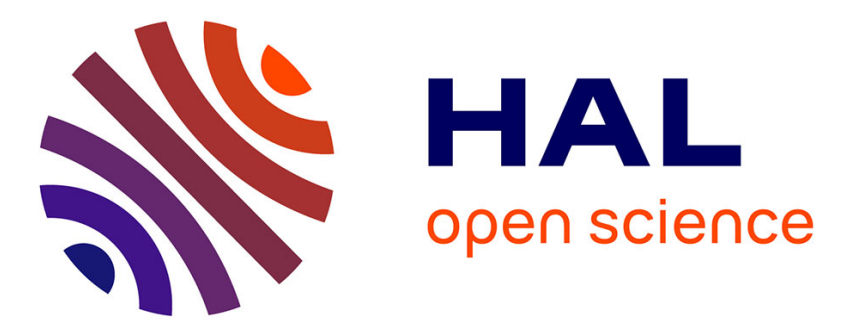

\title{
HREM and WAXS Study of the Structure of Metallic Nanoparticles
}

Marie-José Casanove, Pierre Lecante, Etienne Snoeck, A. Mosset, Christian

Roucau

\section{- To cite this version:}

Marie-José Casanove, Pierre Lecante, Etienne Snoeck, A. Mosset, Christian Roucau. HREM and WAXS Study of the Structure of Metallic Nanoparticles. Journal de Physique III, 1997, 7 (3), pp.505515. 10.1051/jp3:1997138 . jpa-00249592

\section{HAL Id: jpa-00249592 https://hal.science/jpa-00249592}

Submitted on 1 Jan 1997

HAL is a multi-disciplinary open access archive for the deposit and dissemination of scientific research documents, whether they are published or not. The documents may come from teaching and research institutions in France or abroad, or from public or private research centers.
L'archive ouverte pluridisciplinaire HAL, est destinée au dépôt et à la diffusion de documents scientifiques de niveau recherche, publiés ou non, émanant des établissements d'enseignement et de recherche français ou étrangers, des laboratoires publics ou privés. 


\title{
HREM and WAXS Study of the Structure of Metallic Nanoparticles
}

\author{
M.-J. Casanove, P. Lecante $\left(^{*}\right)$, E. Snoeck, A. Mosset and C. Roucau \\ CEMES-LOE, CNRS, 29 rue J Marvig, BP 4347, 31055 Toulouse Cedex, France
}

(Received 6 May 1996, revised 2 October 1996, accepted 13 November 1996)

PACS $6146 .+w$ - Clusters, nanoparticles, and nanocrystalline materials

PACS $6116 \mathrm{Bg}$ - Transmission, reflection and scanning electron microscopy (Including EBIC)

PACS $6110 \mathrm{Eq}$ - X゙-ray scattering (includıng small-angle scattering)

\begin{abstract}
Different samples of fine metal colloids synthesized following an organometallic route were investigated by High Resolution Transmission Electron Microscopy (HREM) and Wide Angle X-rays Scattering (WAXS). Simulations based on models for crystalline and multiply twinned partıcles were used to interpret the observations. Deviations from the bulk structure were clearly observed in the smaller particles and attributed to an effect of dimensions. Combined HREM and WAXS experiments proved to be very efficient in the study of ultrafine particles In partıcular, 55-atoms fcc Pt particles were identıfied.
\end{abstract}

\section{Introduction}

Very small colloids can exhibit original properties, completely different from their properties in the bulk. Metallic clusters, in particular, are well known to present a high catalytic activity. Further developments in fields such as solid-state physics (optics, magnetism) or micro-electronics make desirable the use of mono or bimetallic small particles.

The reactivity and the physical properties of metallic nanoparticles largely depend on their size and dispersion. However, the atomic arrangement inside the clusters also has a great influence and its knowledge is essential to gain a better understanding of their properties. Classical methods of structural characterization such as powder diffractometry or conventional Transmission Electron Microscopy (TEM) reach their limit for nanoscale materials $(<5 \mathrm{~nm})$. Accurate determination of the atomic-scale structure of the particles requires investigations using more appropriate techniques such as High Resolution TEM (HREM) or Wide Angle X-ray Scattering (WAXS)

The metallic particles of interest here lie by their sizes at the limit between the molecular and the solid state. They were synthesized $v \imath a$ an organometallic route using so-called soft conditions. Well dispersed stable particles of platinum, iron and cobalt were thus obtained. Influence of the synthesis conditions on the structural features of these metal colloids was determined by combined HREM and WAXS techniques.

(*) Author for correspondence (e-mail: lecante@cemes.fr)

(C) Les Éditıons de Physique 1997 

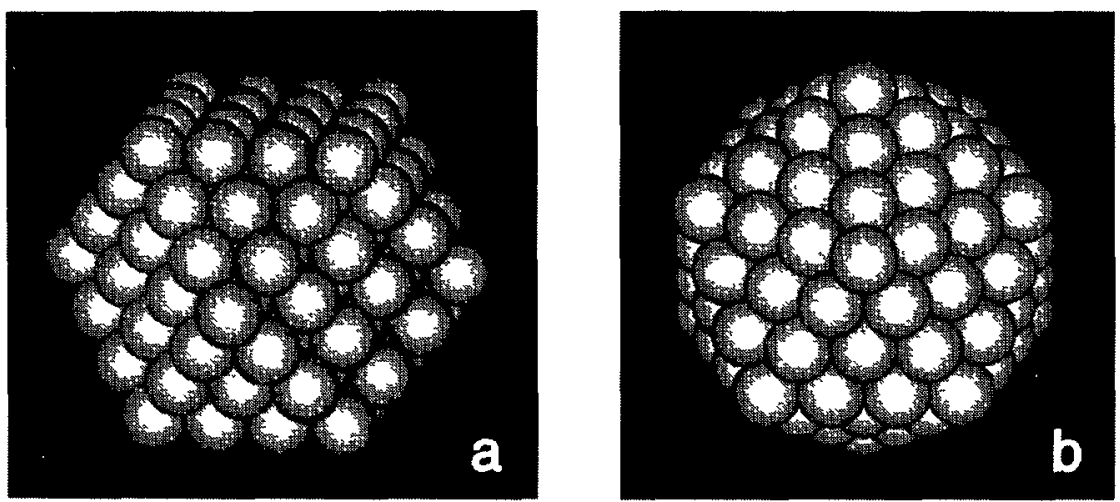

Fig. 1 - Models for 147-atoms closed-shell clusters, a) cuboctahedron; b) icosahedron.

\section{Structural Details}

In the case of close packed cubic or hexagonal (fcc or hcp) metal clusters, a simple model to consider is a super-cell of the bulk lattice From total free energy considerations, it appears that preferred shapes for such particles are closed-shell cuboctahedra obtained through (111) faceting of a basic fcc cube. Since contribution of the surface energy is very high in small colloids, non crystalline, so-called Multiply Twinned. Particles (MTP), models must also be considered [1]. This approach, based on the elasticity theory, introduces essentially two potentially stable or quasi-stable geometries, icosahedra and decahedra [2,3] More recent work based on $a b$ inıtıo calculations led to the same atomic configurations [4]. The models actually used for HREM and WAXS simulations, and presented in Figure 1, are cuboctahedra including $13,55.147, \ldots$ atoms corresponding to the surrounding of a central atom by $1,2,3, \ldots$ complete shells of atoms, and the related icosahedra [5].

\section{Experimental Approach}

All TEM specimens were prepared by slow evaporation of droplets of diluted solutions deposited on a copper grid covered by a very thin holey carbon film (about $2-3 \mathrm{~nm}$ ). The experiments were performed on a Philips CM $30 / \mathrm{ST}$ working at $300 \mathrm{kV}$, whose point resolution is $1.9 \AA$.

A first step in the study of the colloids was the measurement of their size distribution. This was carried out through the numerical analysis of TEM low magnification micrographs. In this procedure, the different particles were first identified according to an upper and lower intensity threshold, then counted and measured. HREM experiments were used to determine the fine structure of the particles. Interplanar angles and spacings were measured from numerical diffractograms (Fourier transforms) of lattice images.

Direct interpretation of the structural features in terms of projected potential is favoured when the particles are observed along suitable directions. For instance lattice images of fcc particles are easily obtained when they are oriented with a [110] or [100] direction parallel to the electron beam. [6]. In the same way, icosahedral particles can be identified by symmetrical considerations when observed along a high-symmetry axis (i.e. five-fold, three-fold or two fold axes). Accurate determination of the geometry of misoriented clusters can however be achieved by image simulation when dealing with particles containıng more than 500 atoms, 
as demonstrated by Kirkland et al. [7] in the case of 923 atoms platinum particles As our particles were very small, comparison between experimental and simulated images was often required even in the case of suitable orientations, particularly for MTP particles. However, when observing ultrafine particles (less than $1 \mathrm{~nm}$ in diameter), the displayed contrast was too low to provide reliable information on either size or structural organization and another approach based on WAXS analysis was chosen to accurately identify these very small clusters.

The diffraction pattern exhibited by materials lacking long-range order can still be considered as a signature of the short- and medium-range structure, since it is a weighted sum of components generated by all interatomic distances inside the sample. The structure-related component of WAXS, the so-called reduced intensity $\imath(s)$ can be examined either directly in the reciprocal space, or more clearly in the real space after Fourier transform [8], provided data collection was performed on a large angular range, and using a radiation of a short enough wavelength (e.g. molybdenum or silver $K_{\alpha}$ radiation) in order to avold excessive termination effects

$$
F(r)=\frac{2 r}{\pi} \int_{s_{\min }}^{s_{\max }} s \imath(s) \sin (r s) \mathrm{d} s
$$

where $s=4 \pi \sin \theta / \lambda$

The experimental reduced Radial Distribution Function (RDF) thus obtained provides important structural informations such as a mean value for the metal-metal bond length, and size estimation for very small particles. A structural model being defined, a theoretical function can be computed through the Debye's formula.

$$
\imath(s)=2 \sum_{\imath=1}^{N-1} \sum_{\jmath=\imath+1}^{N} f_{\imath}(s) f_{\jmath}^{*}(s) \frac{\sin \left(s r_{2 \jmath}\right)}{s r_{\imath \jmath}} \exp \left(-b_{\imath \jmath} s^{2}\right)
$$

where $N$ is the total number of atoms in the model, $f_{2}$ the atomic scattering factor for atom $\imath, r_{\imath \jmath}$ the distance between atoms $i$ and $\jmath . b_{\imath \jmath}$ a dispersion factor affecting the $\imath-j$ interaction In our case, the $b_{\imath}$ factor is defined as the sum of atomic dispersion factors $b_{\imath}$ which can be compared to the $\left\langle u^{2}\right\rangle$ used for crystalline structures.

For regular homogenous objects like metallic particles, the scattering function $i(s)$, or more easily its Fourier transform, can unambiguously be related to a specific geometry (e.g. decahedron or icosahedron, see Fig 2a) and to its extent (Fig. 2b).

For this study, a small amount of powder was sealed, raw from the synthesis, inside a thin-walled Lindemann glass capillary of $1 \mathrm{~mm}$ in diameter. Scattering data were collected on a Enraf-Nonius CAD-4 diffractometer using a zirconium filter in the diffracted beam in order to reduce fluorescence from platinum. Data sets including 460 points equidistant in $s$-space were collected for an empty capillary which was then filled with the fine powder of particles (Fig. 3a). Air scattering was also collected. Despite the high intensity of the empty capillary, its actual contribution to scattering, after correction for the absorption by platinum, is strongly attenuated. The reduced intensity function $i(s)$ was extracted following conventional procedures (Fig. 3b) A small amount of residual fluorescence was removed using a procedure previously described in [9], here adapted for cylindrıcal geometry.

\section{Results and Discussion}

4.1. Cobalt Particles. - Well dispersed very fine cobalt particles were synthezised by decomposition of an organo-metallic precursor dissolved in tetrahydrofurane (THF) in the presence of polyvinylpyrrolidone (PVP) under hydrogen atmosphere at different temperatures [10]. 


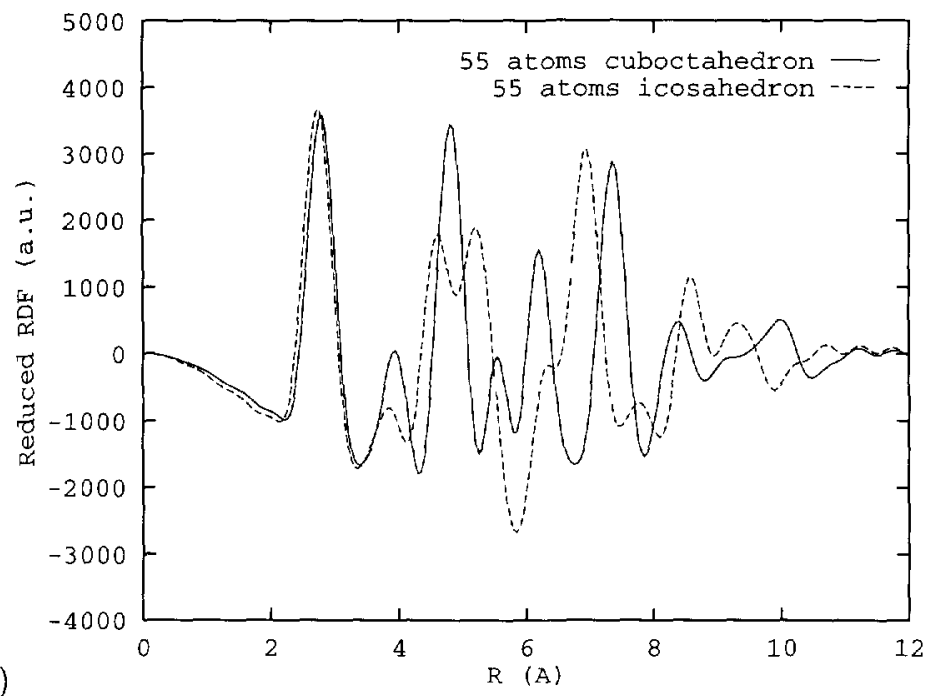

a)

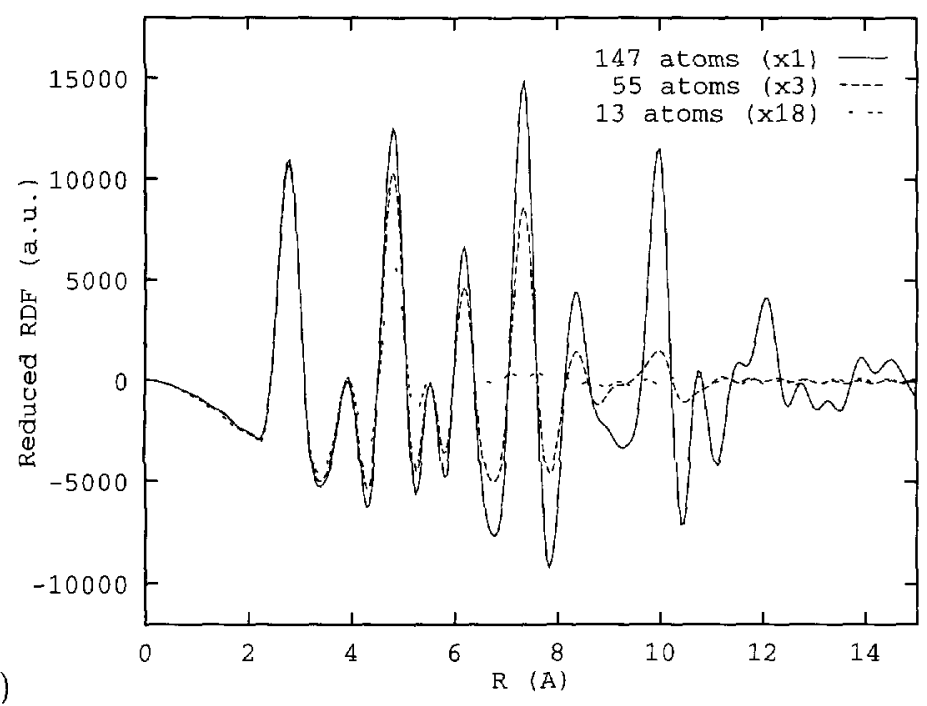

Fig 2. - Dependence of the reduced RDF on a) the structure, b) the size of the particles.

Figure 4 presents a typical histogram of the cobalt particles. It was found that low temperatures of elaboration $\left(0^{\circ} \mathrm{C}\right)$ favoured the formation of smaller particles (mean size $10 \AA$ ) while particles synthesized at $60^{\circ} \mathrm{C}$ had a mean diameter around $25 \AA$. The fcc structure was clearly identified by HREM experiments in particles of mean diameter about $13-15 \AA$ or more. The lattice image of one of these particles is presented in Figure 5 together with its corresponding numerical diffractogram. A large amount of defocus was applied in order to enhance the contrast of the different lattice planes. The angles between the different spots, which correspond to the main spatial frequencies in the lattice image, are indicated in the diffractogram The relative radial distances and angles of the different pairs of spots are in good agreement with the spot diffraction pattern of a fcc crystal observed along a [110] zone axis The occurrence 


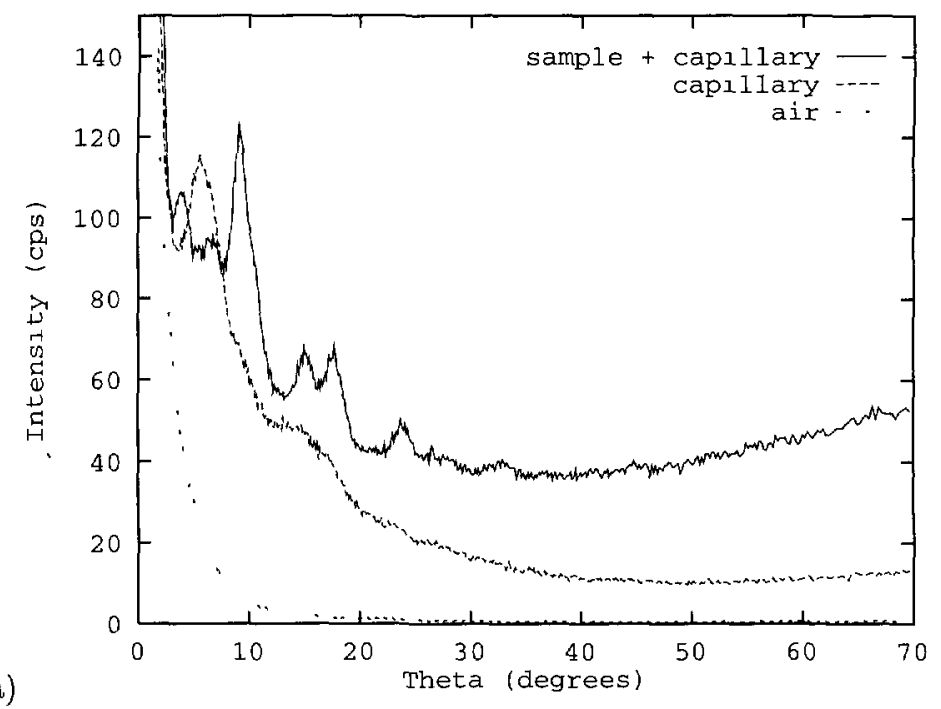

a)

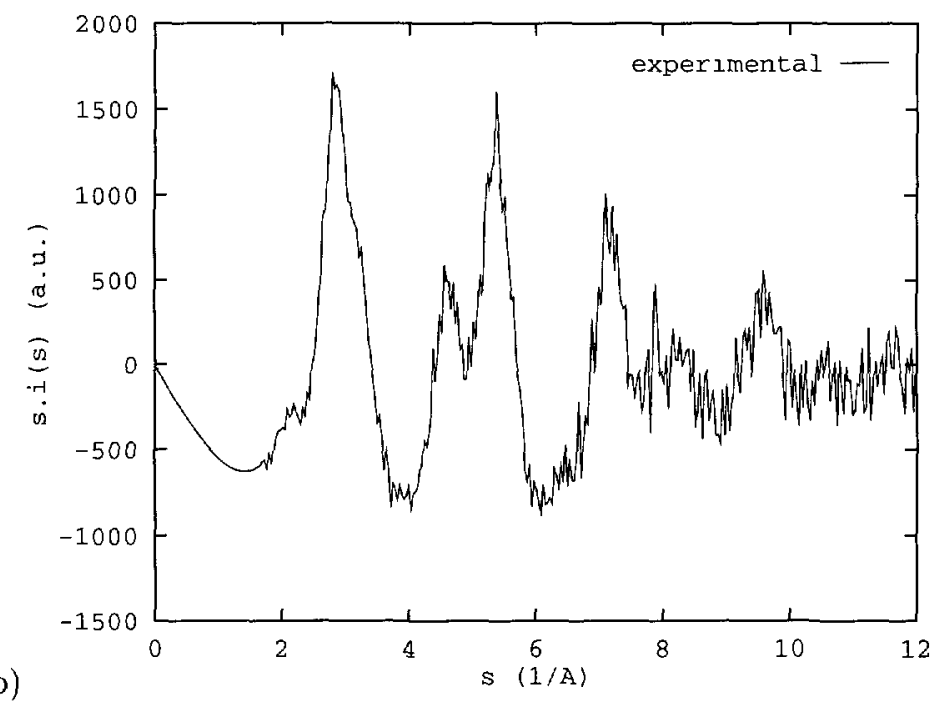

Fig. 3. - Experimental intensity of the Pt collord. a) raw components, b) reduced.

of a close packed fcc structure in metals whose bulk structure is hcp can be considered as the result of an effect of dimensions. Such behaviour was indeed reported in very thin cobalt films in multilayered heterostructures [11]. The presence of different atomic configurations for the smaller particles cannot however be ruled out.

4.2. Iron Particles. - Fe clusters were prepared by sonolysis of a solution of $\mathrm{Fe}(\mathrm{CO})_{5}$ in anisol, in the presence of polydimethylphenyleneoxide (PPO) under Ar or $\mathrm{H}_{2}$ atmosphere. Complete description of the elaboration process can be found in reference [12]. This synthesis leads to the formation of particles with a mean diameter around $30 \AA$, nearly twice the size of the Co particles. Reactions conducted in the presence of hydrogen produced even larger 


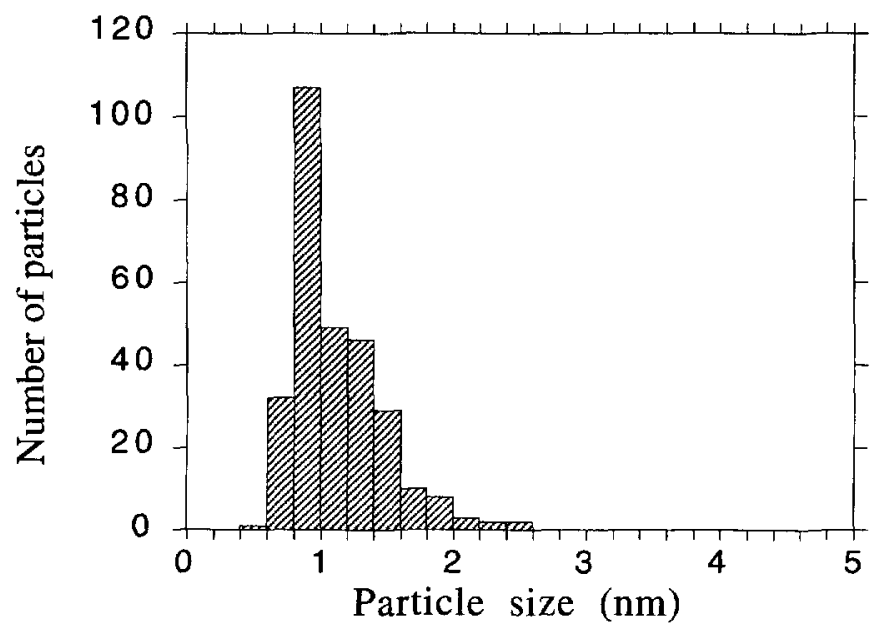

Fig 4 - Size distribution of the Co collord

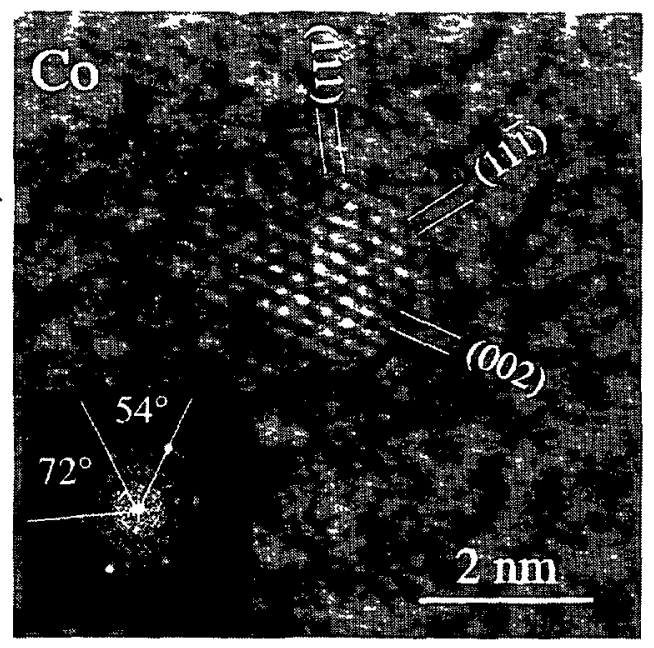

Fig. 5. - $[1 \overline{1} 0]$ zone axis HREM image and corresponding diffractogram (inset) of a $20 \AA$ fcc Co particle. The angular relations between spots are indicated in the diffractogram

particles (around $50 \AA$ ), all exhibiting a fcc structure as evidenced by HREM experiments. The Fe particles synthesized in the presence of argon adopted two different structures. The smaller clusters displayed a bcc structure and the larger ones a fcc structure, as the particles synthesized in the presence of $\mathrm{H}_{2}$. One of the bcc particles, observed along a [111] zone axis, is displayed in Figure 6. The corresponding Fourier transform showed that the three sets of planes had equal spacings and defined angles of $60^{\circ}$. This diffractogram was identified as a [111] zone axis diffraction pattern, the three sets of planes corresponding to (011) type planes One of the known effects of size reduction is to lead to more compact atomic organizations. Accordingly, in the case of iron whose bulk structure is bcc, the fcc structure could be expected in small particles. As the smaller particles observed here display a bcc structure, the presence 


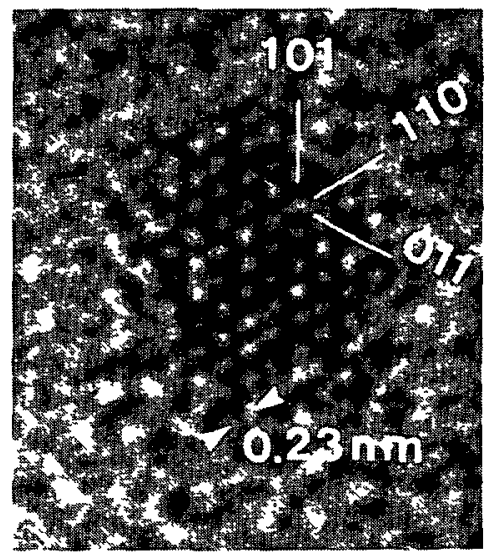

Fig. $6-[\overline{1} 11]$ zone axıs HREM micrograph of a bcc Fe partıcle.

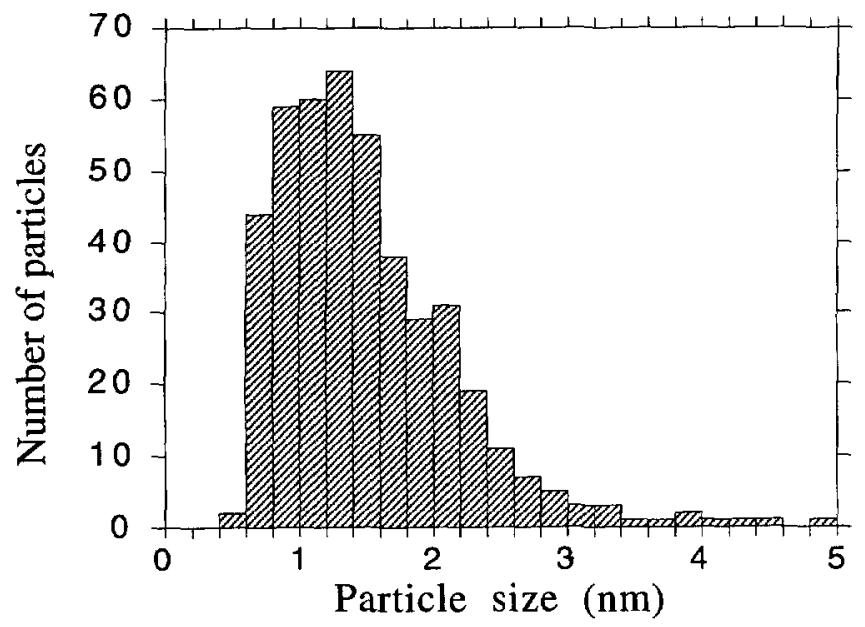

Fig 7 - Size distribution of the Pt collond after redissolution in $\mathrm{CH}_{2} \mathrm{Cl}_{2}$.

of a fcc structure in the larger ones cannot readily be attributed to a dimension effect. This structure may have been stabilized by the incorporation of interstitial carbon atoms or result from localized high temperatures during the sonication process before a rapid quench

4.3. Platinum Particles. - Platinum particles were formed from the reaction of an organometallic precursor with $\mathrm{CO}$ in toluene followed by redissolution in $\mathrm{CH}_{2} \mathrm{Cl}_{2}$ [13]. The size histogram of the colloid thus obtained is presented in Figure 7. HREM experiments combined with diffractograms analysis showed that the structure of the partıcles was fcc, nearly perfect cuboctahedral shapes being commonly observed in the TEM specimens, as confirmed by the very good agreement between the experimental and simulated images ( $c f$. Fig. 8). Further dissolution in THF led to a narrower distribution (Fig. 9). These particles displayed very low contrast and WAXS experiments were preferred to HREM in order to check the atomicscale features. The experimental RDF resulting from the WAXS measurements is presented 

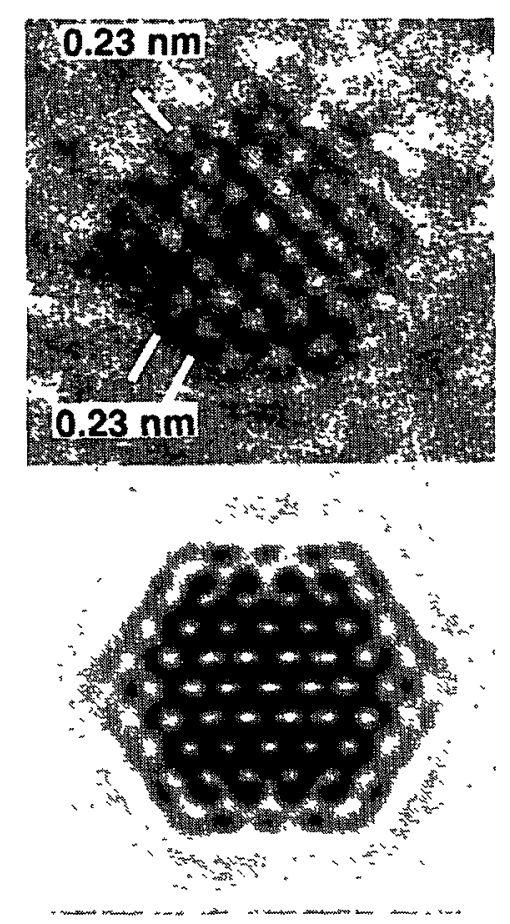

Fig. $8-[110]$ zone axıs HREM experimental and simulated images of a perfect Pt cuboctahedron

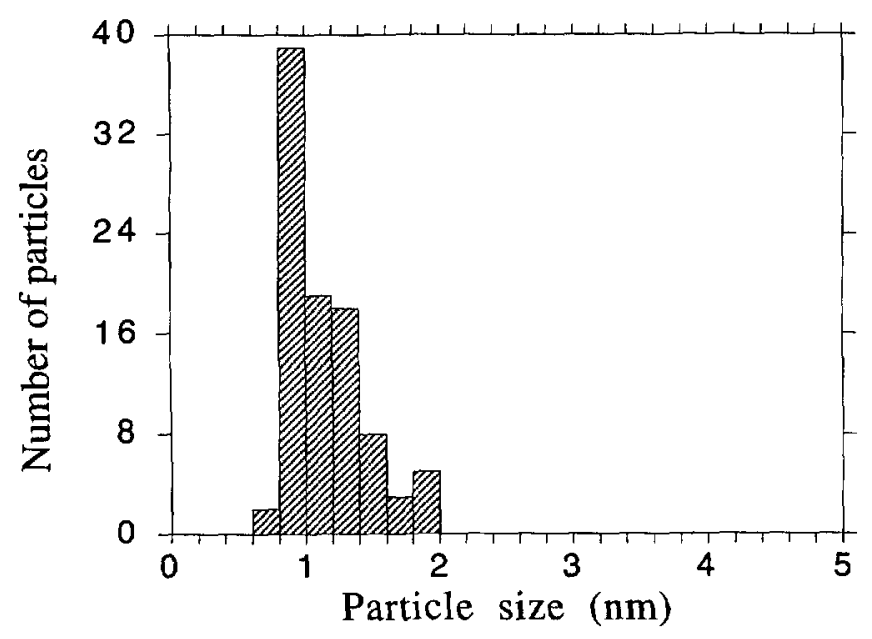

F1g. 9. - Size distribution of the Pt colloid after redissolution in THF

in Figure 10 together with the simulated function. Comparison of experimental data with Figure 2 provides a clear indication that the fcc structure was adopted by these ultrafine particles. The best simulation was obtained for a mixed size model including $90 \%$ of 55 -atoms clusters and $10 \%$ of 147 -atoms clusters, which is in good agreement with the size dispersion 


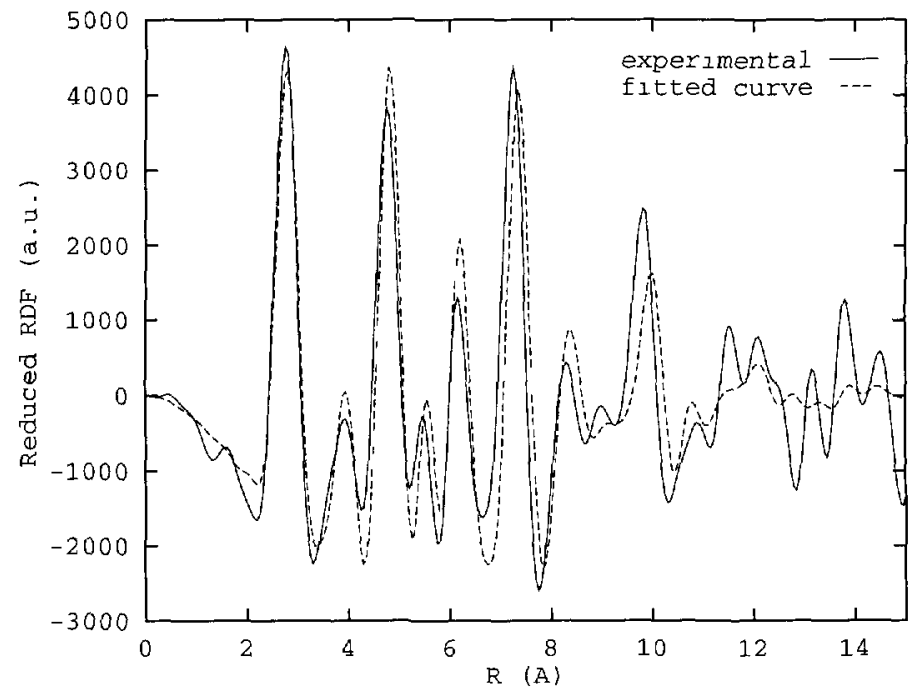

Fig 10. - Experimental and simulated (55-atoms and 147-atoms clusters in a $90 \cdot 10$ ratio) reduced $\mathrm{RDF}$ of the $\mathrm{Pt}$ colloid after redissolution in THF

oberved by TEM. To our knowledge, it is the first time that a fcc structure is demonstrated experimentally in such small clusters. This atomic configuration was proposed for 55-atoms Au clusters by Marcus et al. [14], but although EXAFS experiments prove very powerful to determine accurately the environment of the different atoms, they cannot allow to statue in favour of a particular geometry of clusters. The WAXS experiments showed a contraction of the Pt bulk parameter by $1.5 \%$, which could be attributed to a Gibbs pressure effect. Such a contraction was also observed on the first-neighbour distances in [14].

Addition of a small quantity of $\mathrm{PPh}_{3}$ in the previous colloid caused the loss of the fcc structure, the mean size of the particles remaining the same. No lattice planes could be observed in these samples where the particles were imaged as roughly circular patterns, and a non-crystalline atomic organization was suspected. Indeed, simulated images of a 147-atoms icosahedron compared qualitatively well with experimental images of the bigger particles (about $12 \AA$ ). Comparison between a simulated image of an icosahedron observed along its five-fold symmetry axis and the experimental image of one of the particles is presented in Figure 11.

\section{Conclusion}

Combined HREM and WAXS experiments have proved very efficient to identify the different geometries of ultrafine metal particles. The presence of a fcc structure was evidenced in Co small particles and attributed to a dimensionality effect. On the contrary, this study showed that small particles of bcc metal as iron may keep this structural arrangement. A fcc structure was observed, in our case, in the larger Fe particles. Evidence of the evolution of the mean size and structure of $\mathrm{Pt}$ particles as a function of the redissolutions was given by HREM experiments. WAXS results showed without ambiguity that the ultrafine Pt particles redissolved in THF adopted the fcc cuboctahedron structure. 


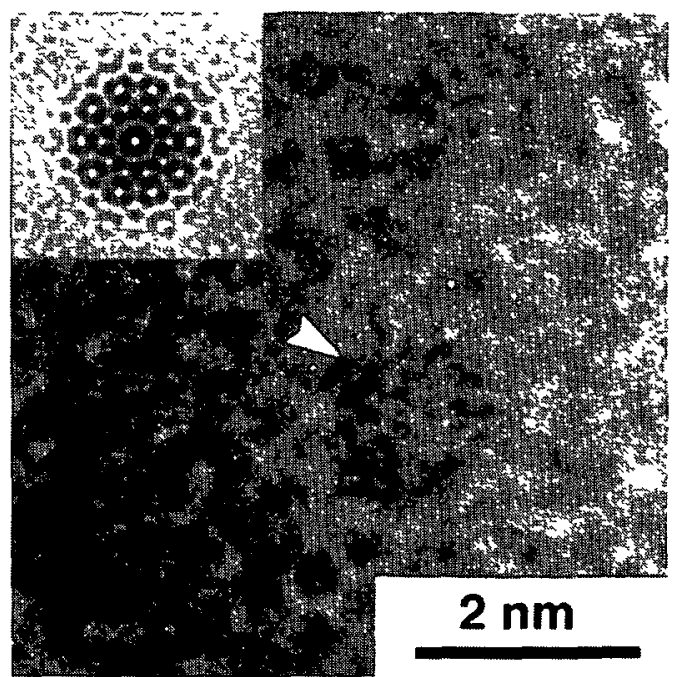

Fig. 11. - Experimental ımage of a non-crystalline Pt particle The displayed pattern is in good agreement with the simulated image of a 147 atoms icosahedron observed along the five-fold symmetry axis (inset)

\section{Acknowledgments}

The authors wish to thank Dr. B. Chaudret group from the "Laboratoire de Chimie de Coordination (LCC)" in Toulouse for providing them with high quality samples

\section{References}

[1] Ino S., Stability of multiply-twinned particles, J. Phys. Soc. Jpn 27 (1969) 941.

[2] Marks L.D., Surface structure and energetics of multiply twinned particles, Philos. Mag. A 49 (1984) 81.

[3] Howie A. and Marks L.D, Elastic strains and the energy balance for multiply twinned particles, Phılos. Mag. A 49 (1984) 95.

[4] Yi J.-Y., Oh D.J. and Bernholc J., Structural distorsions in metal clusters, Phys. Rev. Lett. 67 (1991) 1594.

[5] Mackay A.L., A dense non-crystallographic packıng of equal spheres, Acta Cryst. 15 (1962) 916.

[6] Malm J.-O., HRTEM studies of supported metal particles, Thesis (Lund, Sweden, 1991).

[7] Kirkland A.I., Jefferson D.A., Tang D. and Edwards P.P., High-resolution image simulatıons of small metal particles, Proc. $R$. Soc. Lond A 434 (1991) 279.

[8] Warren B.E., X-ray diffraction (Addison-Wesley Publishing Company, Reading, Mass.. 1969).

[9] Lecante P . Mosset A. and Galy J., Structural studies of amorphous Zn-P films, J. Mater. Scı. 27 (1992) 3286. 
[10] Osuna J., de Caro D., Chaudret B., Snoeck E., Respaud M., Broto J.-M. and Fert A., Synthesis, characterization and magnetic properties of cobalt nanoparticles from an organometallic precursor, J. Phys. Chem. (submitted)

[11] Fontaine A., Baudelet F., Pizzini S, Brouder C., Dartyge E., Giorgetti C., Krill G., Kappler J.P.. Piecuch M., Maurer M., Ravet M.F. and Dupuis V., J. Magn. Magn. Mater. 121 (1993) 10.

[12] de Caro D., Ould Ely T., Mari A. and Chaudret B., Synthesis, characterization and magnetic studies of non agglomerated zerovalent iron particles. Unexpected size dependence of the structure, Chem. Mater. 8 (1996) 1987.

[13] Rodriguez A., Amiens C., Chaudret B., Casanove M.-J , Lecante P. and Bradley S., Synthesis and isolation of cuboctahedral and icosahedral platinum particles Ligand-dependent structures, Chem. Mater 8 (1996) 1978

[14] Marcus M A., Andrews M.P and Zegenhagen J., Structure and vibrations of chemically produced Au55 clusters. Phys. Rev. B 42 (1990) 3312. 